\title{
Comparative Assessment of the Central Vein Pressure by Two Techniques: Centrally and Peripherally Inserted Central Catheter
}

\author{
Siamak Yaghoubi, ${ }^{1}$ Hamid Kayalha, ${ }^{1}$ Ali Mansoori, ${ }^{1}$ Sayed Mehran Marashian, ${ }^{2}$ and Alireza \\ Jahangirifard ${ }^{3, *}$ \\ 1 Velayat Clinical Research Development Unit, Shahid Rajaei Hospital, Qazvin University of Medical Sciences, Qazvin, IR Iran \\ ${ }^{2}$ Chronic Respiratory Disease Research Center, National Research Institute of Tuberculosis and Lung Diseases, National Respiratory Diseases Research Network, Shahid Beheshti \\ University of Medical Sciences, Tehran, IR Iran \\ ${ }^{3}$ Tracheal Diseases Research Center, National Research Institute of Tuberculosis and Lung Diseases (NRITLD), Shahid Beheshti University of Medical Sciences, Tehran, IR Iran \\ ${ }^{*}$ Corresponding author: Alireza Jahangirifard, Tracheal Diseases Research Center, National Research Institute of Tuberculosis and Lung Diseases (NRITLD), Shahid Beheshti University \\ of Medical Sciences, Tehran, IR Iran. Tel:+98-2833760620(669), Fax: +98-2833790611, E-mail: omidjahangiri_55@yahoo.com \\ Received: March 31, 2015; Accepted: May 16, 2015
}

\begin{abstract}
Background: The central venous pressure (CVP) is a reliable indicator used to assess intravascular status. Although this is usually accomplished by placement of a central venous catheter(CVC), there are several situations, when a CVC may be practically difficult or even impossible to be used.

Objectives: The present study aimed to assess the CVP and compare the techniques presently used in this regard, to confirm the peripherally inserted central catheter(PICC), as the default choice in the case of similar findings for CVP measurement. The PICC is usually mounted through a minor surgery, in patients who need CVC.

Patients and Methods: Through a randomized controlled clinical trial, we compared two techniques of CVP measurement, including centrally inserted central catheter(CICC) and PICC, for the patients who were admitted in the intensive care unit, during 2013, at a referral hospital in Tehran, Iran.

Results: There was a strong correlation between CICC and PICC in CVP measurement $(\mathrm{P}<0.001)$, as shown by the Pearson's coefficient $(\mathrm{r}$ $=0.92$ ).

Conclusions: The PICC can be advised in CVP assessment, due to lower complications, along with similar measurement results with CICC. The PICC does not require physician expertise to be set and is also simple to apply. Although there were different measures of CVP, by the used techniques, the strong correlation between the findings could guide us to determine the exact CVP measures, by considering that correlation, as well.
\end{abstract}

Keywords: Central Venous Pressure; Centrally Inserted Central Catheter; Peripherally Inserted Central Catheter; Intensive Care Units

\section{Background}

Central vein cannulation/catheterization (CVC) is a standard technique for central vein pressure (CVP) assessment, particularly in the case of vascular surgery, vasoactive medication administration, or resuscitation by fluid therapy (1). There are several indications for CVC, such as CVP monitoring, pulmonary artery catheterization and study, transvenous cardiac pacing, emergency hemodialysis, medication administration, fast infusion, air emboli aspiration, recurrent blood withdrawal and limited peripheral vascular availability (1). The CVP is a reliable indicator, used to assess intravascular status. Although this is usually accomplished by placement of a CVC, there are several conditions when a CVC may be practically difficult or impossible to be used (2).

Since 1960, when CVC was tried for the first time, the procedure has been performed through the right internal jugular vein, by anesthesiologists (1), while it could be conducted from the left internal jugular (3), subclavian (3), and external jugular veins (1). The peripherally in- serted central catheter (PICC), although is not confirmed to be an acceptable technique to be used in the place of centrally inserted central catheter, would be a suitable alternative $(4,5)$, in the case of long-term parenteral therapy, because it is set on the patient's bedside, with local anesthesia, does not require physician expertise to be settled and has the least complications and scarring. Using an antecubital vein, like the basilic vein, is preferred for PICC, rather than cephalic veins catheterization.

Usually, the CVP measured by PICC is just more than what is reported by CICC, in the same case, although the difference is not significantly significant. When used through the antecubital vein, in abduction position, the tip of the PICC catheter may enter the heart, much deeper than usual, raising the risk of cardiac rupture and arrhythmia. Also, there is a risk of PICC dislocation and its entrance into blood flow, if is accompanied by any other central vein catheter (1).

In operating room, CVCs are used only during the operation, without radiologic imaging for checking the tip

Copyright (C) 2015, Shahid Beheshti University of Medical Sciences. This is an open-access article distributed under the terms of the Creative Commons AttributionNonCommercial 4.0 International License(http://creativecommons.org/licenses/by-nc/4.0/) which permits copy and redistribute the material just in noncommercial usages, provided the original work is properly cited. 
position. The place of the catheter is usually checked by observing blood aspiration through the lumens, in the above setting. This is done while the position of the tip of the catheter has to be radiologically checked after the surgery is finished. The multiport $20-\mathrm{cm}$ seven-French is the most used catheter, in general (1).

The risk of cardiac rupture and/or cardiac tamponade rises if the tip of the catheter is in the heart, or below the horizontal level of the superior vena cava pericardial reflection. The tip would be ideally in the SVC and below the inferior rim of the clavicle, above the third rib, between T4 and T5, the azygos vein, the carina, or in the main right bronchus (1).

In terms of complications, mechanical, thromboembolic, and infective problems account as the main categories of consequences for CICC. Vascular damage, hemothorax, cardiac tamponade, hematoma-induced compressed airways, laryngeal and tracheal trauma, pneumothorax, nerve damages, arrhythmias and mediastinal subcutaneous emphysema are the most known mechanical complications.

Thromboembolic consequences mainly include vein thrombosis, pulmonary emboli, arterial thrombosis and emboli, and catheter/guide wire-induced emboli. Infection at the site of catheterization, septicemia, and endocarditis are often named, between the infective outcomes of CICC (1).

Yun et al. showed no difference between PICC and CICC, in hemodynamic control, among their candidates, for liver transplantation in 2011 (6). The two techniques did not differ in a study by Latham et al. in 2011, when used to measure CVP through PICC, via the basilic veins of left or right upper extremities (7). Likewise, Alansari et al. in 2004, realized that the two techniques had significantly similar results in CVP assessment, although CICC needs physician expertise, while PICC could be set by other staff(8).

This was because consequent pneumothorax, excessive bleeding, neck hematomas, and carotid perforation were less encountered, while the similarity between the two methods in terms of CVP measures was evident. However, due to narrower lumen, while being longer, PICC sounds to report higher measures of CVP than CICC, as Black et al. realized, in 2000 (9).

\section{Objectives}

The present study aimed to assess the CVP and compare the named techniques, in this regard, to use PICC, as the default choice in the case of similar findings, for CVP measurement. The PICC is usually set through a minor surgery, in patients who need CVC.

\section{Patients and Methods}

Through a cross-sectional study, we compared two techniques of CVP measurement, including CICC and PICC, for the patients who were admitted in intensive care unit (ICU), during 2013, at a referral hospital in Tehran, Iran. The patients enrolled in the study were randomly picked from ICU cases with head trauma, considering the inclusion and exclusion criteria. The sample size was determined to a 31 total. Head injury cases, between 20 and 70 years of age, were checked for exclusion criteria, including unknown neck trauma, which needed collar, pulmonary edema, acute respiratory distress syndrome, positive end-expiratory pressure $>5 \mathrm{mmHg}$, and cardiac failure, before getting the informed consent from the families.

The CICC was set through the right internal jugular vein, under a regional anesthesia. The PICC was utilized via the basilic vein, in the forearm, under local anesthesia. All the patients got both techniques for CVP measurement, at the same time. All the vital signs and complications were recorded during the study.

\subsection{Statistics}

Considering a $\mathrm{P}<0.005$ in 95\% confidence interval (CI) for statistical significance, quantitative data were reported, using central tendencies, including the mean, median and their standard deviations, while qualitative data were checked for their frequencies, by chi-square test or Fisher's exact test. Because of only one studied population, for the two techniques, paired t-test was used in quantitative matters.

\subsection{Ethics}

Usually, one of the named techniques is used for CVP measurement in the ICU and the patients are often unconscious. Therefore, the patients' families were asked to give their informed consents, after explaining the aims and process of the present clinical trial.

The participants were free to leave the study, at any stage, and there was no additional cost to pay for setting the second catheter, by the patients or families. The CICC is generally the most used technique, although PICC, which was used additionally, by the current study, has already less complications, as many authors concluded before.

\section{Results}

Totally, 31 patients participated in the present study including 17 (54.9\%) men and 14 (45.1\%) women. There was a strong correlation between CICC and PICC in CVP measurement $(\mathrm{P}<0.001)$, as the Pearson's coefficient showed $(r=0.92)$. This means that our study could not find a significant difference between the two techniques in terms of CVP reported amounts, as can be seen in Table 1 .

Table 1. Comparative Means of Central Venous Pressure Measures by Centrally Inserted Central Catheter and Peripherally Inserted Central Catheter ${ }^{a}$

\begin{tabular}{lcccc}
\hline Complications & Values $^{\text {b }}$ & Mean Difference & T-test & P \\
\hline CICC & $10.9 \pm 1.2$ & 0.2 & 11.14 & 0.09 \\
PICC & $11.1 \pm 1.9$ & & & \\
\hline
\end{tabular}

abbreviations: CICC, Centrally Inserted Central Catheter; PICC, Peripherally Inserted Central Catheter.

${ }^{b}$ Data are presented as mean \pm SD. 
Yaghoubi S et al.

Table 2. Complications of the Procedure of Central Venous Pressure Measurement ${ }^{\mathrm{a}, \mathrm{b}}$

\begin{tabular}{lccccc}
\hline Complications & Pneumothorax & Carotid Perforation & Emphysema & Arrhythmia & Infection \\
\hline CICC & $2(6.5)$ & $2(6.5)$ & $1(3.25)$ & $0(0)$ & $0(0)$ \\
PICC & $0(0)$ & $0(0)$ & $0(0)$ & $0(0)$ & $0(0)$ \\
\hline
\end{tabular}

a Abbreviations: CICC, Centrally Inserted Central Catheter; PICC, Peripherally Inserted Central Catheter.

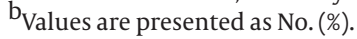

Concerning the complications, pneumothorax was seen in two cases (6.5\%), which as the consequences of CICC. As Table 2 shows, carotid perforation similarly occurred in two patients (6.5\%). Emphysema was found only in one (3.25\%) case. There was no complication related to PICC, such as local infection or arrhythmia and so on.

\section{Discussion}

The present study focused on the CVP measures and the occurrence of complications of CICC and PICC and found that there is no difference between them in CVP measures, although CICC complications, like pneumothorax and carotid perforation, as well as subcutaneous emphysema, were seen, although no PICC-induced problems were reported. Less complications and simple application, besides no need for medical specialist expertise, usually make PICC more applicable in many settings.

There was a correlation between the findings of the techniques, CICC and PICC, with a Pearson's coefficient, just less than 1. This is in line with Yun's performance, in 2011, in South Korea, which was tried among liver transplantation candidates (6). In contrast to Black(9), who realized that the two factors of narrow lumen and long size made the CVP measures higher by PICC, we found similar measures to what Latham and colleagues reported, in 2012, in the SA (7). Separately, similar findings for the CVP measurement, using CICC and PICC, were also reported by Alansari et al. (8), in 2004. In 2009, Tobias explained a study on 18 patients, which assessed CVP measures by peripheral IV cannula (PVP) that is used in PICC. The results showed a $13 \pm 4 \mathrm{mmHg}$ difference between PICC and CICC, in this regard (10), in $11 \%$ of cases, although only 2 $\pm 1 \mathrm{mmHg}$ in the rest of patients, which was significant. Through posterior spine surgery in 40 patients, the mean PVP was $17.55 \pm 4.93 \mathrm{mmHg}$, which was also significantly higher than CVP $(15.52 \pm 4.77 \mathrm{mmHg})$, as reported by Memtsoudis et al. (11). The difference between the measures was 10 times ours. The Pearson's coefficient, in their work, was also similar to ours, for the correlation between PICC and CICC findings. Likely, with the rather lower measures by CICC and PICC than what we found, Choi et al. reported a $1.16 \mathrm{mmHg}$ difference between the mean measures, by the two techniques, with a high correlation $(r=0.89)$ (4). They concluded that PVP could be an alternative to predict CVP, because of the strong correlation, as other authors like Cox et al. realized before.

Through the current trial, we aimed to achieve a conclusion to make a decision to either use PICC, instead of
CICC, or not. Therefore, we tried to assess the main objective of the techniques, as CVP measurement and the complications resulting from them. Regarding complications, CICC had its problems, including pneumothorax, carotid perforation and subcutaneous emphysema, in a total of five cases (16\%). On the contrary, PICC recorded no complications and left CICC behind, in this regard.

To sum up, PICC can be advised in CVP assessment, due to lower complications rate, along with similar measurement results, with CICC. The PICC does not need physician expertise to be set in place and is also simple to apply. Although there are different measures of CVP, by the used techniques, the strong correlation between the findings could guide us to determine the exact CVP measures by considering that correlation, as well.

\section{Footnotes}

Authors' Contributions:Conception and design, Drafting of the article, Final approval of the article, Administrative, technical, or logistic support: Siamak Yaghoubi and Alireza Jahangirifard. Analysis and interpretation of the data, Critical revision of the article for important intellectual content, Statistical expertise, Collection and assembly of data: Hamid Kayalha, Ali Mansoori, Sayed Mehran Marashian.

Financial Disclosure:Qazvin University of medical sciences.

Funding/Support:Qazvin University of medical sciences.

\section{References}

1. Miller RD. Miller's anesthesia. Philadelphia, PA: Churchill Livingstone/Elsevier; 2010. p. 1285

2. Cox P, Johnson JO, Tobias JD. Measurement of central venous pressure from a peripheral intravenous catheter in the lower extremity. South Med J. 2005;98(7):698-702. doi: 10.1097/01. SMJ.0000152543.10264.08. [PubMed: 16108238]

3. Lakhal K, Ferrandiere M, Lagarrigue F, Mercier C, Fusciardi J, Laffon M. Influence of infusion flow rates on central venous pressure measurements through multi-lumen central venous catheters in intensive care. Intensive Care Med. 2006;32(3):460-3. doi: 10.1007/s00134-005-0049-6. [PubMed: 16477417]

4. Choi SJ, Gwak MS, Ko JS, Kim GS, Kim TH, Ahn H, et al. Can peripheral venous pressure be an alternative to central venous pressure during right hepatectomy in living donors? Liver Transpl. 2007;13(10):1414-21. doi:10.1002/lt.21255. [PubMed:17902127]

5. McLemore EC, Tessier DJ, Rady MY, Larson JS, Mueller JT, Stone $\mathrm{WM}$, et al. Intraoperative peripherally inserted central venous catheter central venous pressure monitoring in abdominal aortic aneurysm reconstruction. Ann Vasc Surg. 2006;20(5):577-81. doi:10.1007/s10016-006-9108-x. [PubMed:16871437]

6. Yun JY, Park SH, Cho DS, Jeung HJ, Lee SA, Seo SJ. Comparison of 
the central venous pressure from internal jugular vein $\&$ the pressure measured from the peripherally inserted antecubital central catheter in liver transplantation recipients . Korean J Anesthesiol. 61(4):281-7. [PubMed: 22110879]

7. Latham HE, Rawson ST, Dwyer TT, Patel CC, Wick JA, Simpson SQ. Peripherally inserted central catheters are equivalent to centrally inserted catheters in intensive care unit patients for central venous pressure monitoring. J Clin Monit Comput. 2012;26(2):8590. doi:10.1007/s10877-012-9337-1. [PubMed:22290064]

8. Alansari M, Hijazi M. Central venous pressure from peripherally inserted central catheters correlates well with that of centrally inserted central catheters. Chest. 2004;126(4):873S.

9. Black IH, Blosser SA, Murray WB. Central venous pressure Mea- surement : Peripherally inserted Catheters Versus Centrally Inserted Catheters. Crit Care Med. 2000;28(12):3833-6. [PubMed: 11153622]

10. Tobias JD. Measurement of central venous pressure from a peripheral intravenous catheter in the prone position during spinal surgery. South Med J. 2009;102(3):256-9. doi: 10.1097| SMJ.ob013e3181971614. [PubMed: 19204622]

11. Memtsoudis SG, Jules-Elysse K, Girardi FP, Buschiazzo V, Maalouf D, Sama AA, et al. Correlation between centrally versus peripherally transduced venous pressure in prone patients undergoing posterior spine surgery. Spine (Phila Pa 1976). 2008;33(18):E643-7. doi:10.1097/BRS.0b013e31817c6c2f. [PubMed: 18708916] 